University of New Hampshire

University of New Hampshire Scholars' Repository

Physics Scholarship

Physics

$1-1-1994$

\title{
Empirical modeling of the quiet time nightside magnetosphere
}

\author{
A. T. Y. Lui \\ Harlan E. Spence \\ Boston University, harlan.spence@unh.edu \\ D. P. Stern
}

Follow this and additional works at: https://scholars.unh.edu/physics_facpub

Part of the Physics Commons

\section{Recommended Citation}

Lui, A. T. Y., H. E. Spence, and D. P. Stern (1994), Empirical modeling of the quiet time nightside magnetosphere, J. Geophys. Res., 99(A1), 151-157, doi:10.1029/93JA02647.

This Article is brought to you for free and open access by the Physics at University of New Hampshire Scholars' Repository. It has been accepted for inclusion in Physics Scholarship by an authorized administrator of University of New Hampshire Scholars' Repository. For more information, please contact Scholarly.Communication@unh.edu. 


\title{
Empirical modeling of the quiet time nightside magnetosphere
}

\author{
A. T. Y. Lui \\ Applied Physics Laboratory, The Johns Hopkins University, Laurel, Maryland
}

H. E. Spence

Space and Environment Technology Center, The Aerospace Corporation, Los Angeles, California

\author{
D. P. Stern
}

Laboratory for Extraterrestrial Physics, NASA Goddard Space Flight Center, Greenbelt, Maryland

Empirical modeling of plasma pressure and magnetic field for the quiet time nightside magnetosphere is investigated. Two models are constructed for this study. One model, referred to here as T89R, is basically the magnetic field model of Tsyganenko (1989) but is modified by the addition of an inner eastward ring current at a radial distance of $\sim 3 R_{E}$ as suggested by observation. The other is a combination of the T89R model and the long version of the magnetic field model of Tsyganenko (1987) such that the former dominates the magnetic field in the inner magnetosphere, whereas the latter prevails in the distant tail. The distribution of plasma pressure, which is required to balance the magnetic force for each of these two field models, is computed along the tail axis in the midnight meridian. The occurrence of pressure anisotropy in the inner magnetospheric region is also taken into account by determining an empirical fit to the observed plasma pressure anisotropy. This effort is the first attempt to obtain the plasma pressure distribution in force equilibrium with magnetic stresses from an empirical field model with the inclusion of pressure anisotropy. The inclusion of pressure anisotropy alters the plasma pressure by as much as a factor of $\sim 3$ in the inner magnetosphere. The deduced plasma pressure profile along the tail axis is found to be in good agreement with the observed quiet time plasma pressure for geocentric distances between $\sim 2$ and $\sim 35 R_{E}$.

\section{INTRODUCTION}

Although the magnetosphere has been studied and surveyed extensively for over 3 decades since the advent of spacecraft, there are still outstanding tasks pertaining to the quiet time magnetosphere. One of these is a quiet time magnetospheric model with specification of the magnetic field configuration and the associated equilibrium plasma pressure distribution. In the quiet time Earth's magnetosphere, the inertial force $\rho d \mathbf{v} / d t$ is usually insignificant, and therefore force balance should exist between magnetospheric plasma pressure and electromagnetic forces. An explicit configuration resembling the magnetosphere and satisfying force balance has yet to be devised.

Equilibrium of the magnetosphere has been studied theoretically in the two limiting situations of the inner magnetosphere, where the Earth's dipole field dominates, and the magnetotail, where the field arises mainly from the theta-shape tail current system. The inclusion of the transition region between these two simplified situations has proved difficult [Voigt and Wolf, 1988]. In the inner magnetosphere, Sckopke [1972] studied equilibrium under the assumption of isotropic plasma pressure in a perturbed dipole field. This type of investigation was recently ex-

Copyright 1994 by. the American Geophysical Union.

Paper number 93JA02647.

0148-0227/94/93JA-02647\$05.00 tended by Zavriyev and Hasegawa [1989] and Cheng [1992] with the more realistic consideration of anisotropy in plasma pressure as observed [e.g., Lui and Hamilton, 1992]. The magnetotail equilibrium has been studied analytically [Harris, 1962; Kan, 1973] as well as examined by numerical simulation [Toichi, 1972; Birn, 1989; Hesse and Birn, 1992]. Unfortunately, no global three-dimensional equilibrium solutions are known. Present global MHD simulations do not stabilize enough to provide them. Two-dimensional numerical solution, when convection is included, appears to evolve in ways that may preclude a steady state equilibrium [Erickson and Wolf, 1980; Erickson, 1984; Hau et al., 1989], although this may be merely an artifact of the two-dimensional limitation [Kivelson and Spence, 1988].

An alternative approach to obtaining equilibrium configurations is to use data. For example, the magnetic field models of Tsyganenko [1987, 1989], which give averaged magnetic field values satisfying the static Maxwell's equations, have been used extensively in relating magnetospheric regions to the ionospheric level and vice versa [Elphinstone et al., 1990; Stasiewicz, 1991; Elphinstone and Hearn, 1992; Pulkkinen et al., 1992]. However, early models of this type were badly out of force balance [Walker and Southwood, 1982]. Particularly pertinent questions related to any empirical magnetic field model are therefore the following:

(1) What is the plasma pressure distribution in force equilibrium with the empirical field model?

(2) How is this plasma pressure distribution compared with actual measurements? 
It is quite evident that the availability of plasma pressure distribution in force balance with the magnetic forces in an empirical field model will further enhance its utilization.

Spence et al. [1987, 1989] and Kan et al. [1992] have inverted the earlier magnetic field models [Tsyganenko and Usmanov, 1982; Tsyganenko, 1987] to obtain the equilibrium plasma pressure along the tail axis at midnight by assuming isotropic or nearly isotropic plasma. The goal of this paper is to extend these previous studies in deriving empirical magnetic field models for the quiet time magnetosphere, with the associated plasma pressure in force equilibrium with the model magnetic field and in good agreement with observations. Two features are incorporated, namely, the presence of an eastward inner ring current and the occurrence of anisotropic plasma pressure. These two features are found to be essential in arriving at a realistic plasma pressure profile. This study treats the simple situation in which the dipole tilt angle is zero, making the configuration symmetric and easy to handle without significantly altering the results.

\section{The Modified Magnetic Field Modets}

The magnetic field models of Tsyganenko [1987, 1989], hereafter referred to as T87L (long version) and T89, have a number of features that lend themselves to easy applications. The magnetic field in these models is given in an analytical form, with most terms bearing physical insights on the various current systems contributing to the total magnetic field at a given location. The coefficients of these terms are obtained through a least-squares fit to actual measurements and are derived separately for different levels of the $K p$ index to allow for the variability of the magnetic field with geomagnetic activity. Since we consider here the quiet time magnetosphere, the activity level chosen for this study corresponds to the $K p=0$ level. Modified versions of these field models form the basis from which magnetic field models are constructed here.

The ring current is dominated by the gradient of plasma pressure. At the inner edge of the ring current, the plasma pressure decreases rapidly inward, producing an eastward flowing ring current in the inner magnetosphere [ $L u i$ and Hamilton, 1992]. This ring current is featured in neither the T87L nor the T89 model. Although this eastward flowing ring current may not alter the magnetic field to a great extent, as will be demonstrated later, its absence tremendously affects the plasma pressure distribution required to maintain equilibrium with the magnetic forces. Without such an eastward flowing current, the plasma pressure in force equilibrium with the magnetic field will have to increase monotonically inward, as indicated by the earlier work of Spence et al. [1989] and Kan et al. [1992], and is contrary to the observed plasma pressure distribution. Even though the required volume current density for the eastward ring current is small, the $\mathbf{j} \times \mathbf{B}$ force is significant, since the field strength is high (about inversely proportional to the third power of the geocentric distance) in the inner magnetosphere.

To include the inner ring current in the model, we construct its vector potential using a procedure similar to the one employed in the T89 model to represent the outer ring current, i.e.,

$$
A_{\mathrm{IRC}}=C_{\mathrm{IRC}} \rho S_{\mathrm{IRC}}^{-3}
$$

where $C_{\text {IRC }}$ is a coefficient controlling the intensity of the inner ring current and

$$
\begin{gathered}
S_{\mathrm{IRC}}=\sqrt{\rho^{2}+\left(a_{\mathrm{IRC}}+\xi_{\mathrm{IRC}}\right)^{2}}, \\
\xi_{\mathrm{IRC}}=\sqrt{z^{2}+\left(D_{\mathrm{o}}+\gamma_{\mathrm{IRC}} h_{\mathrm{IRC}}\right)^{2}}, \\
h_{\mathrm{IRC}}=0.5\left[1+x\left(x^{2}+L_{\mathrm{IRC}}\right)^{-1 / 2}\right] .
\end{gathered}
$$

The location of the point in space is given in cylindrical coordinates by $(\rho, \phi, z)$ and in Cartesian coordinates by $(x$, $y, z)$. The physical significance of the nonlinear parameters is the same as given for the outer ring current by Tsyganenko [1989]. The radial scale length $a_{\text {IRC }}$ relates to the geocentric distance for the inner ring current. The half thickness of the inner ring current region is represented by $D_{0}$. The variation of this thickness with local time, i.e., the difference between dayside and nightside, is controlled by the function $h_{\mathrm{IRC}}$ with its scale length $L_{\mathrm{IRC}}$. We find that the flow reversal of the ring current from westward to eastward inside $L \approx 3.5$ can be reproduced reasonably well by adopting the following values for the above parameters: $C_{\text {IRC }}=760 \mathrm{nT}, a_{\text {IRC }}=1.5 R_{E}, D_{0}=1.8 R_{E}, \gamma_{\text {IRC }}=-0.2723$ (same as the value for the quiet time outer ring current), and $L_{\mathrm{TRC}}=1.5 R_{E}$. To keep the total magnetic moment of the ring currents the same as the original ring current in the T89 model, we have modified the outer ring current strength by

$$
C_{\mathrm{RRC}}=C_{\mathrm{ORC}}-C_{\mathrm{IRC}} \times\left(a_{\mathrm{IRC}} / a_{\mathrm{ORC}}\right)^{2},
$$

where the subscripts RRC and ORC denote, respectively, the revised and original outer ring current parameters in T89. It turns out that the modification is extremely slight (only $\sim 0.2 \%$ ). This model with the inner eastward ring current and a correspondingly modified outer ring current strength is identified hereafter as T89R.

Since the T89 and T89R field models give rather weak magnetic fields in the magnetotail [Stern and Tsyganenko, 1992; Rostoker and Skone, 1993; Peredo et al., 1993], we have also explored the T87L field model. To take advantage of the fact that the T89R model is better than the T87L model in the inner magnetospheric region, but the reverse is true for the distant tail [Tsyganenko, 1989; Donovan et al., 1992], we combine the two field models by constructing a hybrid field model (designated hereafter as TH) with a transition parameter $\epsilon$ such that

$$
\begin{gathered}
B_{z, \mathrm{TH}}=\epsilon B_{2,87 \mathrm{~L}}+(1-\epsilon) B_{z, 89 \mathrm{R}}, \\
\epsilon=0.5-0.5 \sin \left(\left(2\left(x-x_{\mathrm{L}}\right) /\left(x_{\mathrm{U}}-x_{\mathrm{L}}\right)-1\right) \pi / 2\right),
\end{gathered}
$$

where $B_{z}$ is the $z$ component of the magnetic field along the midnight meridian and subscripts $87 \mathrm{~L}$ and $89 \mathrm{R}$ denote parameters from the T87L and T89R models, respectively. The parameters $x_{\mathrm{L}}$ and $x_{\mathrm{U}}$ are the lower and upper bounds of $x$ for the transition. The transition parameter $\epsilon$ is 0 at $x=x_{U}$ and 1 at $x=x_{\mathrm{L}}$, with a smooth derivative $d \epsilon / d x$ at the end points. The boundary points adopted here are $x_{U}$ 
$=-5 R_{E}$ and $x_{\mathrm{L}}=-25 R_{E}$. We note that the region of interest in this paper is the equatorial plane along the tail axis in which the magnetic field has only the $z$ component (for a dipole tilt angle of $0^{\circ}$ ). The divergence of the magnetic field along the tail axis therefore reduces to $\partial B_{\mathrm{z}} / \partial z$. It is clear then that the linear combination of the magnetic fields from the T87L and T89R models as expressed in (6) is also divergence free along the tail axis. On the other hand, if this hybrid model were to be extended beyond the tail axis, there would be a small nonzero divergence if the other field components were not modified accordingly.

Figure $1 a$ shows the profiles of magnetic field values in logarithmic scale from the four magnetic field models, i.e., $\mathrm{T} 87 \mathrm{~L}, \mathrm{~T} 89, \mathrm{~T} 89 \mathrm{R}$, and $\mathrm{TH}$, at the equatorial region along the midnight meridian. Note that the four magnetic field profiles are quite different between $x \approx-8 R_{E}$ and $-30 R_{E}$. The highest magnetic field values come from T87L, followed by TH, T89, and T89R. Although the inclusion of the inner ring current only slightly weakens the magnetic field, this effect is manifested quite dramatically in the extremely low field region of the T89 model, where the field magnitude goes below $1 \mathrm{nT}$.

The volume current densities from these four models at the equatorial region along the midnight meridian are examined in Figure $1 b$. The T87L model typically gives the lowest current densities among these models. Tailward of $x=-10 R_{E}$, the T89 and T89R models give almost the same values, whereas the TH model shows noticeably lower current densities between $x \approx-10$ and $-30 R_{E}$. The lower values from the $\mathrm{TH}$ model, in comparison with the T89 and T89R models, are expected from the influence of the T87L model. Earthward of $x \approx-10 R_{E}$, although the current densities from the T89R and TH models are almost

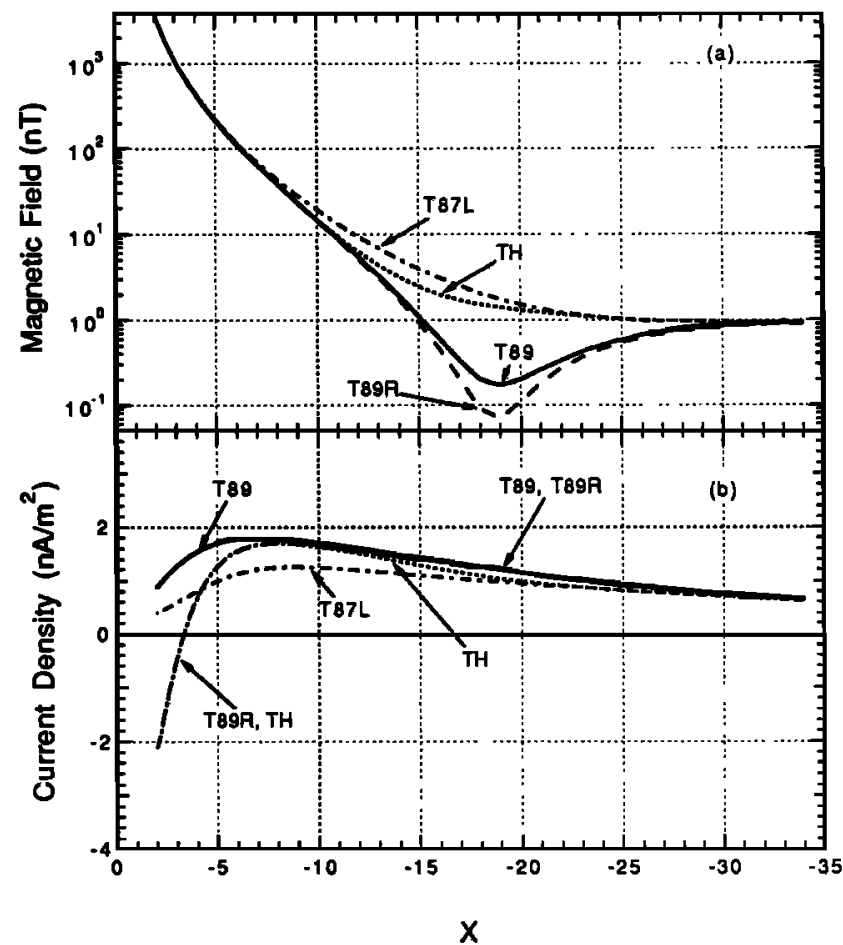

Fig. 1. (a) Magnetic field profiles along the midnight meridian at the equatorial plane from four magnetic field models. (b) Current density profiles along the midnight meridian at the equatorial plane from four magnetic field models. identical, those from the T89 and T87L models have different values. Earthward of $x \approx-8 R_{E}$, both the T89 and T87L models show a persistent westward current. In contrast, the T89R and TH models show a reversal of westward ring current to an eastward ring current at $\sim 3.5 R_{E}$. The presence of this current reversal is modeled to represent the reversal noted from the CCE observations [Lui and Hamilton, 1992]. In comparison, we find the observed current densities appear to be higher than the model values at the current density peak but smaller than the model values at distances further downstream. In addition, the observed current densities are more variable, reflecting the filamentary nature of current much like the magnetotail current [McComas et al., 1986].

The observed anisotropy of plasma pressure taken from Lui and Hamilton [1992] is shown in Figure 2 together with an empirical fit. The anisotropy is seen to be large in the inner region and decreases rather systematically at distances further downstream. The empirical fit to the anisotropy is a fifth-order polynomial function of $x^{-1}$, i.e.,

$$
\begin{aligned}
P_{\perp} / R_{\|}-1= & a_{0}+a_{1} x^{-1}+a_{2} x^{-2}+a_{3} x^{-3} \\
& +a_{4} x^{-4}+a_{5} x^{-5},
\end{aligned}
$$

with coefficients $a_{0}=-0.410554, a_{1}=-9.94369, a_{2}=$ -86.9877, $a_{3}=-504.066, a_{4}=-1110.73$, and $a_{5}=$ -847.912 . The range of validity for this fit is $-2.5 R_{E}>x$ $>-15 R_{E}$ such that the plasma pressure is isotropic at $x$ $\approx-15 R_{E}$. That the pressure should be isotropic downstream of $x \approx-15 R_{E}$ is indicated by the studies of Stiles et al. [1978] and Baumjohann and Paschmann [1989].

Given a magnetic field model, it is straightforward to compute the magnetic force $\mathbf{j} \times \mathbf{B}$ and determine the pressure force required to balance it. The radial profile of perpendicular plasma pressure can be obtained by integrating

$$
\frac{\partial P_{\perp}}{\partial r}=\left[\mathbf{j} \times \mathbf{B}+\left(P_{\perp}-P_{\|}\right)(\mathbf{b}: \nabla) \mathbf{b}\right]_{r},
$$

where the subscript $r$ indicates the radial component of the vector quantity, $\mathbf{b}$ is the unit magnetic field vector $\mathbf{B}, \mathrm{j}$ is the volume current density, and $P_{\perp}$ and $P_{\|}$are the plasma pressure components perpendicular and parallel to the observed magnetic field direction, respectively.

Figure $3 a$ shows the result from this computation for the T89R model with the plasma pressure at $x=-34 R_{E}$ taken to be $0.074 \mathrm{nPa}$. This initial value of plasma pressure is obtained by computing the plasma pressure re-

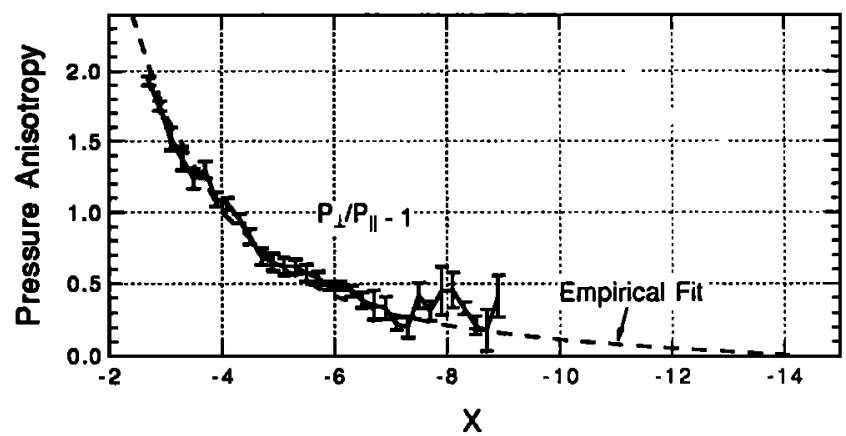

Fig. 2. Observed pressure anisotropy profile of the nightside magnetosphere and an empirical fit. 


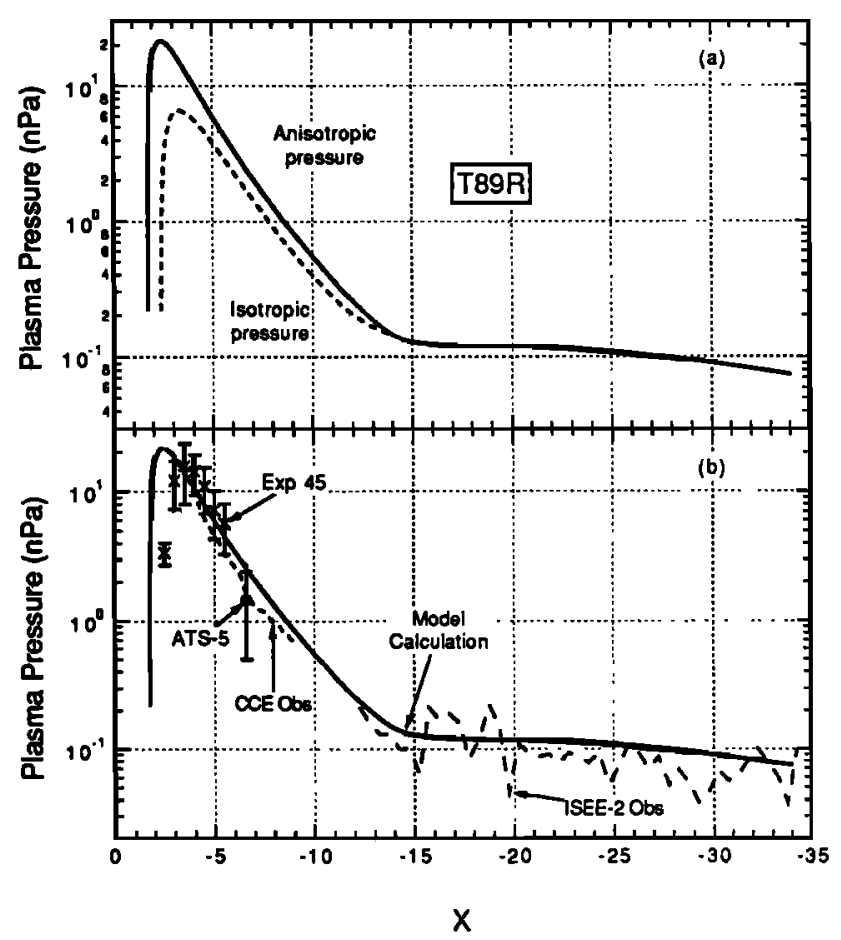

Fig. 3. (a) Equatorial profiles of the perpendicular plasma pressure along the midnight meridian inverted from the T89R magnetic field model assuming isotropic plasma pressure (dashed trace) and adopting the pressure anisotropy shown in Figure 2 (solid trace). (b) Equatorial profile of the perpendicular plasma pressure along the midnight meridian inverted from the T89R magnetic field model in comparison with measured values of perpendicular plasma pressure from several satellites.

quired at the neutral sheet $(z=0)$ to balance the "asymptotic" magnetic field value for the tail lobe region of the T89R model at that distance. The "asymptotic" magnetic field value is the $x$ component of the field at $z=20 R_{E}$, which in this case is $\sim 13.6 \mathrm{nT}$. It is worth noting that since the plasma pressure is shown in logarithmic scale, a larger initial value for the plasma pressure will not affect the plasma pressure in the inner magnetosphere significantly. The plasma pressure is assumed isotropic at $x=-15 R_{E}$ and further downstream. The computed profile of the perpendicular plasma pressure is given by the solid trace. For comparison, the profile of plasma pressure, assuming pressure isotropy at all distances, is given by the dashed trace. It is quite apparent that the assumption of isotropic pressure results in an underestimate of the plasma pressure at the inner magnetosphere by as much as a factor of $\sim 3$. This finding can readily be understood by noting that the plasma pressure anisotropy inside $x \approx-15 R_{E}$ for the quiet time magnetosphere is in the sense of $P_{\perp}>P_{\|}$. With this sense of pressure anisotropy, the second term on the right of equation (9) adds to the first one, thus requiring a larger pressure gradient for force equilibrium, as pointed out earlier by Lui and Hamilton [1992].

The computed perpendicular plasma pressure profile is compared with observations from Explorer 45, ATS 5, ISEE 2 , and CCE in Figure $3 b$. The ISEE 2 data are taken from Spence et al. [1989] for the $K p=-1$ condition, the CCE data from Lui and Hamilton [1992], the ATS-5 data from DeForest and McIlwain [1971], and the Explorer 45 data from Spence et al. [1989], using measurements of 1- to
872-keV protons presented by Smith and Hoffman [1973]. The range of pressure values from Explorer 45 data indicated by the error bars actually spans the observed pressure taken during geomagnetically quiet to disturbed conditions, and thus the lower limit should be used for comparison with the model calculation. As can be seen from Figure $3 b$, the model pressure profile is in general above the observed pressure profile, especially in the outer region. This finding is understandable and is to be expected because the observed pressure is not necessarily obtained right at the equatorial plane and thus is expected to underrepresent the actual observed pressure at the equatorial plane. Inside $4 R_{E}$, the pressure determined from Explorer 45 is noticeably lower than that from CCE values and the model calculation. This difference may be due to the upper energy threshold being only $872 \mathrm{keV}$ for Explorer 45 measurements, whereas that of CCE extends to $>4 \mathrm{MeV}$. It is possible that a significant contribution to plasma pressure inside $4 R_{E}$ comes from particles above the $872-\mathrm{keV}$ level. We have evaluated this possibility using several midnight passes of CCE during quiet conditions. We found the ions with energy of $>872 \mathrm{keV}$ contributing typically $\sim 50-65 \%$ to the plasma pressure at geocentric distances inside $4 R_{E}$. Since the disparity in the determination of pressure between Explorer 45 and CCE is about a factor of 6 , we conclude that the different energy passbands can account for a significant portion, but not the entirety, of the observed pressure difference.

Overall, we find very good agreement exists between the observed and computed profiles of plasma pressure on the basis of the T89R model. Another notable feature of the model pressure profile is its relative constancy between the downstream distances of 16 and $25 R_{E}$. This finding indicates the small magnitude of the $\mathbf{j} \times \mathbf{B}$ force in this region. Reexamining Figure 1 reveals that the main reason for the decrease in the $\mathbf{j} \times \mathbf{B}$ force in the T89R model is the small value of the magnetic field in that region (Figure 1a), since the volume current density is still substantial at those distances (Figure $1 b$ ). The more abrupt change in the radial profile of plasma pressure occurs in the downstream distances between $x \approx-12 R_{E}$ and $x \approx$ $-15 R_{E}$, as noted earlier by Spence et al. [1989]. The $\mathbf{j} \times$ $B$ force is therefore relatively large at the transition region between the dipolelike and taillike field configurations. It is interesting to note that this region may correspond to the hinging point of the tail, where the tangential stress acted on the magnetotail by the solar wind at the tail magnetopause is balanced by the attractive force between the Earth's dipole and the tail current system [Siscoe and Cummings, 1969].

A similar plasma pressure profile can be obtained from the TH model, as shown in Figures $4 a$ and $4 b$. The plasma pressure based on the formula from Spence and Kivelson [1993],

$$
P(\mathrm{nPa})=89 e^{-0.59|x|}+8.9|x|^{-1.53}
$$

is also shown for comparison; the downstream distance $x$ is in Earth radii. The assumption of isotropic pressure gives an underestimate of plasma pressure by as much as a factor of $\sim 3$ in the inner magnetosphere as before. The most significant difference between the two anisotropic pressure profiles in Figures 3 and 4 lies in the downstream 


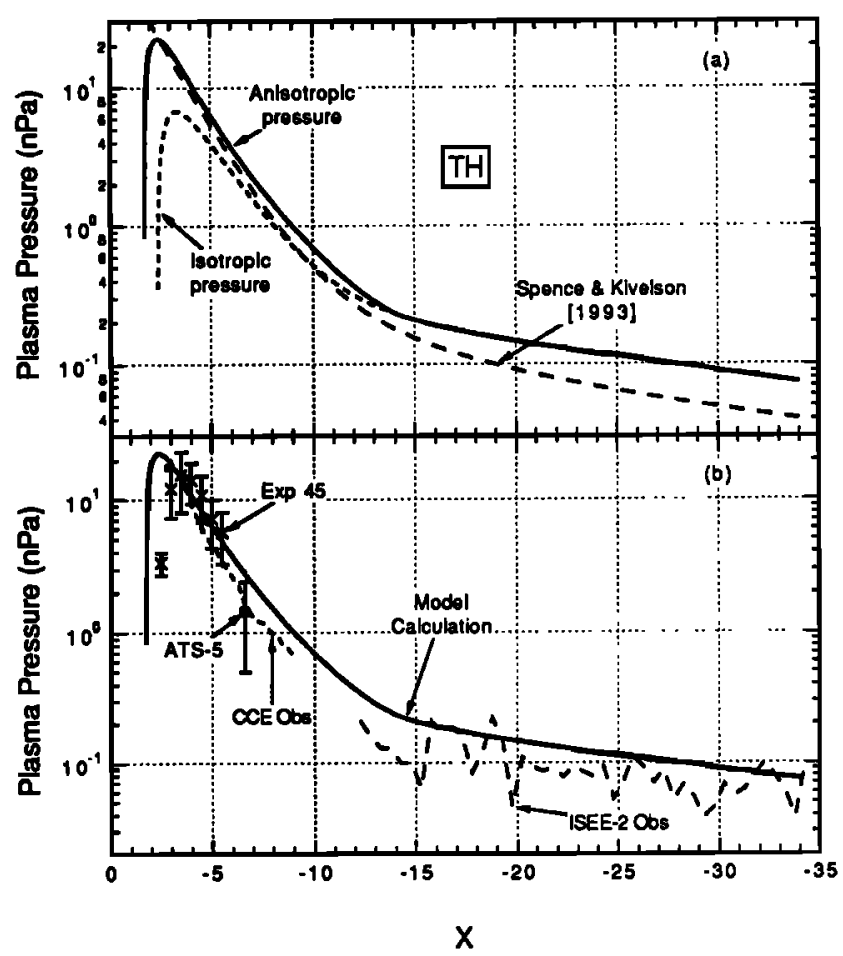

Fig. 4. (a) Similar to Figure $3 a$ except that the perpendicular plasma pressure is inverted from the TH magnetic field model instead. (b) Similar to Figure $3 b$ except that the perpendicular plasma pressure is inverted from the TH magnetic field model instead.

region of $x \approx-10$ to $-20 R_{E}$. Whereas the T89R profile shows a relatively constant pressure in this region, the other profile shows a continuous pressure increase toward the Earth. This continuous rise, however, only contributes rather insignificantly to the plasma pressure earthward of $x \approx-8 R_{E}$. The continuous change in plasma pressure reflects a more gradual transition between the dipolelike and taillike field configurations as well as a more $x$-dependent pressure for the near-Earth tail region in this model than that in the T89R model. The empirical formula by Spence and Kivelson [1993] also compares reasonably well with the model profile. The formula gives a good fit to the plasma pressure within the range of $x \approx-2.5$ to $-15 R_{E}$ but overestimates it inside this region and underestimates it further tailward.

\section{Summary AND Discussion}

We have investigated two empirical models for the specification of magnetic field and the plasma pressure for the quiet time nightside magnetosphere. The derived magnetic field models are extensions of the previous magnetic field models of Tsyganenko [1987, 1989] by the addition of an inner eastward ring current. Furthermore, in constructing the equilibrium plasma pressure along the tail axis in the midnight meridian, we have taken into account the presence of plasma pressure anisotropy in the inner magnetosphere earthward of $x \approx-15 R_{E}$. An empirical fit to the observed pressure anisotropy in the inner magnetosphere (between 2.5 and $15 R_{E}$ ) is also provided. These two new features profoundly affect the deduced plasma pressure profile. The first feature leads to an earthward reduction of the plasma pressure in the innermost part of the mag- netosphere (geocentric distances of $<\sim 3 R_{E}$ ) as observed. The second feature leads to an appropriate evaluation of the plasma pressure gradient. Without the consideration of plasma pressure anisotropy, the deduced plasma pressure may be underestimated by as much as a factor of $\sim 3$. The derived perpendicular plasma pressure is found to be in good agreement with the observed values from ISEE 2, CCE, ATS 5, and Explorer 45. The T89R and TH models therefore provide quite realistic representations along the tail axis in the midnight meridian of not only the quiet time magnetic field but also the associated equilibrium plasma pressure distributions needed to provide the force balance with the magnetic forces in these models. The range of validity for the T89R and TH models in representing the magnetic field, the volume current density, and the anisotropic plasma pressure considered here is $-2.5 R_{E}>x>$ $-35 R_{E}$. The present result also supports the earlier findings from Spence et al. [1989] that the gradient of plasma pressure shows a relatively large change in the transition region between dipolelike and taillike field configurations.

A region called the inner edge of the plasma sheet has been introduced in the early studies of the energetic particle environment in the magnetosphere [Vasyliunas, 1968; Frank, 1971]. It is a region characterized by an exponential decrease of electron energy density with decreasing radial distance and is generally located at geocentric distances of $\sim 6$ to $8 R_{E}$ in the nightside. Many researchers implicitly assume that the inner edge of the plasma sheet is associated with an earthward decrease of the total plasma pressure, and theories on the closure of the large-scale region 2 field-aligned current system [Iijima and Potemra, 1978] have been built upon this assumption (see, for example, the review by Mauk and Zanetti [1987]). As pointed out by Mauk and Zanetti [1987], the observed plasma pressure shows a persistent earthward increase rather than a decrease in the geocentric distances usually ascribed to the inner edge of the plasma sheet. In terms of total plasma pressure, there is no edge at those distances. The plasma commonly identified as constituting the plasma sheet in the outer magnetosphere (tailward of the geocentric distance of $\sim 10 R_{E}$ ) gradually becomes the hot ring current particle population. The absence of a large-scale plasma pressure decrease in the so-called inner edge of the plasma sheet indicates the necessity to modify the conventional mechanism for closing the large-scale region 2 field-aligned current. An alternative means for the region 2 field-aligned current closure in an earthward increasing plasma pressure condition has been proposed by Lui and Hamilton [1992]. They have suggested, based on the work of Sato and Iijima [1979], that the correct sense of field-aligned current will be generated if the $\nabla P_{\perp}$ vector is inclined toward the midnight meridian more than the $\nabla B$ vector in both the premidnight and the postmidnight sectors, since the sense of the field-aligned current is determined by the sign of the triple product of $B, \nabla P_{\perp}$, and $\nabla B$. Figure 5 illustrates this configuration graphically. In other words, a plasma pressure in the midnight sector slightly enhanced over that of the adjacent local time sectors will satisfy the requirement.

At least two aspects of this study can be improved in the future. One is to extend the calculation to other local times besides midnight, and the second is to extend it in the $z$ direction to cover the region away from the equato- 
Generation of the Region II Field-Aligned Current

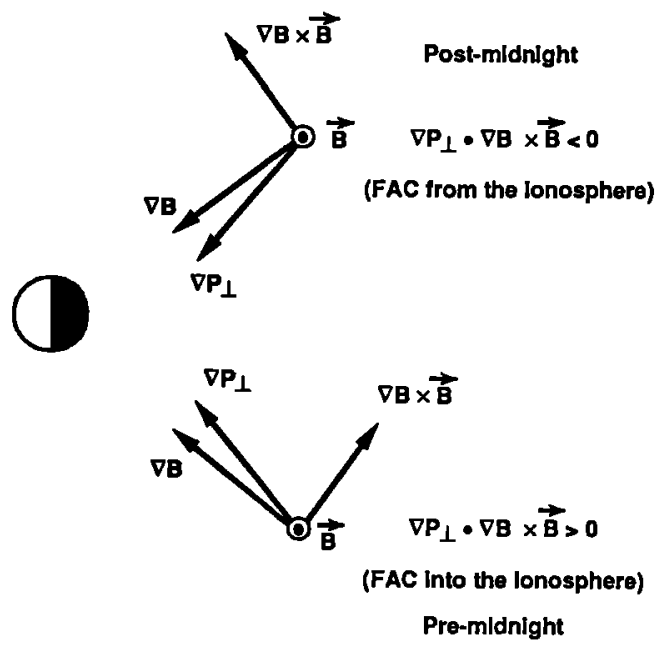

Fig. 5. A schematic diagram to illustrate the relative orientation of the vectors $\nabla B$ and $\nabla P_{\perp}$ to give the correct sense of the fieldaligned current system for region 2 .

rial plane. The former extension requires a better assessment of the variation of pressure anisotropy as a function of local time, which is not presently available. The latter extension requires integrating the variations of both the perpendicular and parallel components of the plasma pressure in a plane. If these extensions were to be incorporated in the TH model, one must also address the extension of the divergence-free property of the magnetic field along the tail axis to other regions in the magnetosphere, which can be accomplished by considering a linear combination of the vector potentials of the T87L and T89R models instead of their magnetic fields. These improvements will be the subject of a future investigation.

Acknowledgments. We would like to thank N. A. Tsyganenko for providing his earlier magnetic field models on which the new models are built. This work was supported by the Atmospheric Sciences Section of the National Science Foundation (Grant ATM9114316 to the Johns Hopkins University Applied Physics Laboratory). The work at The Aerospace Corporation was supported by the Space Physics Program of the National Aeronautics and Space Administration under Grant NAGW-3353.

The Editor thanks T. Pulkkinen and R. H. Comfort for their assistance in evaluating this paper.

\section{REFERENCES}

Baumjohann, W., and G. Paschmann, Determination of the polytropic index in the plasma sheet, Geophys. Res. Lett., 16, $295,1989$.

Birn, J., Three-dimensional equilibria for the extended magnetotail and the generation of field-aligned current sheets, J. Geophys. Res., 94, 252, 1989.

Cheng, C. Z., Magnetospheric equilibrium with anisotropic pressure, J. Geophys. Res., 97, 1497, 1992.

Donovan, E. F., G. Rostoker, and C. Y. Huang, Region of negative $B_{z}$ in the Tsyganenko 1989 model neutral sheet, $J$. Geophys. Res., 97, 8697, 1992.

Elphinstone, R. D., and D. J. Hearn, Mapping of the auroral distribution during quiet times and substorm recovery, Eur. Space Agency Spec. Publ., ESA SP 335, 13, 1992.

Elphinstone, R. D., K. Jankowska, J. S. Murphree, and L. L. Cogger, The configuration of the auroral distribution for interplanetary magnetic field $B_{z}$ northward, 1 , IMF $B_{x}$ and $B_{y}$ dependencies as observed by the Viking satellite, $J$. Geophys. Res., 95, 5791, 1990.
Erickson, G. M., On the cause of X-line formation in the nearEarth plasma sheet: Results of adiabatic convection of plasma sheet, in Magnetic Reconnection in Space and Laboratory Plasmas, Geophys. Monogr. Ser., vol. 30, edited by E. W. Hones, Jr., p. 296, AGU, Washington, D.C., 1984.

Erickson, G. M., and R. A. Wolf, Is steady state convection possible in the Earth's magnetosphere?, Geophys. Res. Lett., 7, $897,1980$.

Frank, L. A., Relationship of the plasma sheet, ring current, trapped boundary, and plasmapause near the magnetic equator and local midnight, J. Geophys. Res., 76, 2265, 1971.

Harris, E. G., On a plasma sheath separating regions of oppositely directed magnetic field, Nuovo Cimento, 23, 116, 1962.

Hau, L. N., R. A. Wolf, G. H. Voigt, and C. C. Wu, Steady state magnetic field configurations for the Earth's magnetotail, $J$. Geophys. Res., 94, 1303, 1989.

Hesse, M., and J. Birn, Three-dimensional MHD modeling of magnetotail dynamics for different polytropic indices, $J$. Geophys. Res., 97, 3965, 1992.

Kan, J. R., On the structure of the tail current sheet, J. Geophys. Res., 78, 3773, 1973

Kan, J. R., W. Sun, and W. Baumjohann, A hybrid equation of state for the quasi-static central plasma sheet, Geophys. Res. Lett., 19, 421, 1992.

Iijima, T., and T. A. Potemra, Large-scale characteristics of fieldaligned currents associated with substorms, J. Geophys. Res., $83,599,1978$.

Kivelson, M. G., and H. E. Spence, On the possibility of quasistatic convection in the quiet magnetotail, Geophys. Res. Lett., $15,1541,1988$.

Lui, A. T. Y., and D. C. Hamilton, Radial profiles of quiet time magnetospheric parameters, J. Geophys. Res., 97, 19,325, 1992.

Mauk, B. H., and L. J. Zanetti, Magnetospheric electric fields and currents, Rev. Geophys., 25, 541, 1987.

McComas, D. J., C. T. Russell, R. C. Elphic, and S. J. Bame, The near-Earth cross-tail current sheet: Detailed ISEE 1 and 2 case studies, J. Geophys. Res., 91, 4287, 1986.

Peredo, M., D. P. Stern, and N. A. Tsyganenko, Are existing magnetospheric models excessively stretched?, J. Geophys. Res., 98, 15,343, 1993

Pulkkinen, T. I., R. J. Pellinen, H. E. J. Koskinen, H. J. Opgenoorth, J. S. Murphree, V. Petrov, A. Zaitzev, and E. Friis-Christensen, Auroral signatures of substorm recovery phase: A case study, in Magnetospheric Substorms, Geophys. Monogr. Ser., vol. 64, edited by J. R. Kan, et al., p. 333, AGU, Washington, D.C., 1992.

Rostoker, G., and S. Skone, Magnetic flux mapping considerations in the auroral oval and the Earth's magnetotail, $J$. Geophys. Res., 98, 1377, 1993.

Sato, T., and T. Iijima, Primary sources of large-scale Birkeland currents, Space Sci. Rev., 24, 347, 1979.

Sckopke, N., A study of self-consistent ring current models, Cosmic Electrodynamics, 3, 330, 1972.

Siscoe, G. L., and W. D. Cummings, On the cause of geomagnetic bays, Planet. Space Sci., 17, 1795, 1969.

Smith, P. H., and R. A. Hoffman, Ring current particle distributions during the magnetic storms of December 16-18, 1971, J. Geophys. Res., 78, 4731, 1973.

Spence, H. E., and M. G. Kivelson, Contributions of the lowlatitude boundary layer to the finite width magnetotail convection model, J. Geophys. Res., 98, 15,847, 1993.

Spence, H. E., M. G. Kivelson, and R. J. Walker, Static magnetic field models consistent with nearly isotropic plasma pressure, Geophys. Res. Lett., 14, 872, 1987.

Spence, H. E., M. G. Kivelson, R. J. Walker, and D. J. McComas, Magnetospheric plasma pressures in the midnight meridian: Observations from 2.5 to $35 R_{E}$, J. Geophys. Res., 94, 5264, 1989.

Stasiewicz, Polar cusp topology and position as a function of interplanetary magnetic field and magnetic activity: Comparison of a model with Viking and other observations, J. Geophys. Res., 96, 15,789, 1991.

Stern, D. P., and N. A. Tsyganenko, Uses and limitations of the Tsyganenko field models, Eos Trans. AGU, 73, 489, 1992.

Stiles, G. S., E. W. Hones, S. J. Bame, and J. R. Asbridge, Plasma sheet pressure anisotropies, J. Geophys. Res., 83, 3166, 1978. 
Toichi, T., Two-dimensional equilibrium solution of the plasma sheet and its application to the structure of the tail magnetosphere, Cosmic Electrodynamics, 3, 81, 1972.

Tsyganenko, N. A., Global quantitative models of the geomagnetic field in the cislunar magnetosphere for difference disturbance levels, Planet. Space Sci., 35, 1347, 1987.

Tsyganenko, N. A., A magnetospheric magnetic field model with a warped tail current sheet, Planet. Space Sci., 37, 5, 1989.

Tsyganenko, N. A., and A. V. Usmanov, Determination of the magnetospheric current system parameters and development of experimental geomagnetic field models based on data from IMP and HEOS satellites, Planet. Space Sci., 30, 985, 1982.

Voigt, G.-H., and R. A. Wolf, Quasi-static magnetospheric MHD processes and the "ground state" of the magnetosphere, Rev. Geophys., 26, 823, 1988.

Walker, R. J., and D. J. Southwood, Momentum balance and flux conservation in model magnetospheric magnetic fields, $J$. Geophys. Res., 87, 7460, 1982.
Vasyliunas, V. M., A survey of low-energy electrons in the evening sector of the magnetosphere with Ogo 1 and Ogo 3, J. Geophys. Res., 73, 2839, 1968.

Zavriyev, A, and A. Hasegawa, The equilibrium dayside magnetosphere, J. Geophys. Res., 94, 10,039, 1989.

A. T. Y. Lui, Applied Physics Laboratory, The Johns Hopkins University, Johns Hopkins Road, Laurel, MD 20723.

H. E. Spence, Space and Environment Technology Center, The Aerospace Corporation, Los Angeles, CA 90045.

D. P. Stern, Laboratory for Extraterrestrial Physics, NASA Goddard Space Flight Center, Greenbelt, MD 20771.

(Received April 15, 1993;

revised July 12, 1993;

accepted September 3, 1993.) 